\title{
Definitions of $\mathrm{BCK}$ algebras and $\mathrm{BCl}$ algebras Zhang Kun-Long ${ }^{a}$, Song Li-xia ${ }^{b}$
}

Department of Basic Science North China Institute of Science and Technology Beijing, 101601, China

azhk1007@126.com, ${ }^{b}$ Songlx100@sina.com

Keywords:BCK algebra; $\mathrm{BCl}$ algebra; Algebra of type(2,0).

Abstract. In this paper, we consider the definitions of the BCK and BCI Algebra. We put out four examples in order to prove that any one of the four conditions in the definition of BCK algebra cannot be proofed by other three conditions. Next we simplify the definitions of the BCK and BCI Algebra by giving new equivalent conditions.

\section{Introduction}

The study of BCK-algebras was initiated by Y. Imai and K. Iséki [4] in 1966 as a generalization of the concept of settheoretic difference and propositional calculus. Since then a great deal of literatures have been produced on the theory of BCK-algebras. In particular, emphasis seems to have been put on the ideal theory of BCK-algebras. The hyper structure theory (called also multialgebras) was introduced in 1934 by F. Marty [1] at the 8th congress of Scandinavian Mathematicians. Around 40 `s, several authors worked on hyper groups, especially in France, United States, Italy, Greece and Iran.

\section{Preliminaries}

Definition 1. An algebra $(\mathrm{A}, *, 0)$ of type $(2.0)$ is called a BCI-algebra if it satisfies the following conditions for $\forall x, y, z \in A$
(1) $((x * y) *(x * z)) *(z * y)=0$
(2) $x * 0=x$
(3) $x * y=0$ and $y * x=0$ imply $x=y$

Definition 2. An algebra $(A, *, 0)$ of type (2.0) is called a BCK-algebra if it satisfies the following conditions for $\forall x, y, z \in A$ :

(1) $((x * y) *(x * z)) *(z * y)=0$

(2) $x * 0=x$

(3) $0 * x=0$

(4) $x * y=0$ and $y * x=0$ imply $x=y$

Hence the BCK-algebra is derived from the BCI-algebra by adding the condition $0 * x=0$. The following theorems show the relation between BCK-algebra and BCI-algebra[2],[3],[5].

Theorem 1. [6] An algebra $(A, *, 0)$ of type $(2,0)$ is a BCK-algebra if and only if there is a partially ordering $\leq$ on A such that the following conditions

hold: for any $x, y, z \in A$ :

(1) $(x * y) *(x * z) \leq(z * y)$,

(2) $x *(x * y) \leq y$,

(3) $0 \leq x$,

(4) $x * y=0$ if and only if $x \leq y$.

Theorem 2. A BCI-algebra is a BCK-algebra if it satisfies the following condition:

$$
x *(x * y)=y *(y * x), \forall x, y \in A
$$


Theorem 3. A BCI-algebra is a BCK-algebra if it satisfies the following condition:

$$
x *(y * x)=x, \forall x, y \in A
$$

Theorem 4. A BCI-algebra is a BCK-algebra if it satisfies the following condition:

$$
(x * y) * y=(x * z) *(y * z), \forall x, y, z \in A
$$

Theorem 5. A BCI-algebra is a BCK-algebra if it satisfies the following condition:

$$
(x * y) *(y * x)=x * y, \forall x, y \in A
$$

Theorem 6. A BCI-algebra is a BCK-algebra if it satisfies the following condition:

$$
(x * y) *((x * y) *(y * x))=0, \forall x, y \in A
$$

Theorem 7. [6] Let $x, y, z \in A$ be any elements in BCK-algebra A. Then

(1) $x \leq y$ implies $x * z \leq y * z$ and $z * x \leq z * y$,

(2) $(x * y) * z=(x * z) * y$,

(3) $x * y \leq z$ if and only if $x * z \leq y$,

(4) $x *(x *(x * y))=x * y$,

(5) $0 *(x * y)=(0 * x) *(0 * y)$,

(6) $(x * y) * x=0$,

(7) $x *(x * y)=0$ if and only if $x=x * y$,

(8) $x, y \leq x *(x * y)$.

The following examples support that the four conditions in the BCK-algebra definition are independent. Therefore, in order to simplify the BCK-algebra definition we have to propose the equivalent new definition of BCK-algebra.

Example 1. Let $X=\{0,1\}$ and $*$ is given by the table 1 .

Table 1.

\begin{tabular}{|l|l|l|}
\hline$*$ & 0 & 1 \\
\hline 0 & 0 & 1 \\
\hline 1 & 1 & 0 \\
\hline
\end{tabular}

Table 2 .

\begin{tabular}{|l|l|l|l|}
\hline$*$ & 0 & 1 & 2 \\
\hline 0 & 0 & 1 & 0 \\
\hline 1 & 1 & 0 & 2 \\
\hline 2 & 2 & 1 & 0 \\
\hline
\end{tabular}

Then $X$ is a BCI-algebra, but not a BCK-algebra. Because it doesn’t satisfy the condition (3) of the BCK definition, that is $0 * 1=1 \neq 0$.

Example 2. Let $X=\{0,1,2\}$ and $*$ is given by the table 2 .

It satisfies the condition (2) (3) (4) of the BCK definition, but doesn't the condition (1).

Example 3. Let $X=\{0,1,2\}$ and $*$ is given by the table 3 .

Table 3.

\begin{tabular}{|l|l|l|l|}
\hline$*$ & 0 & 1 & 2 \\
\hline 0 & 0 & 0 & 0 \\
\hline 1 & 1 & 0 & 2 \\
\hline 2 & 2 & 0 & 0 \\
\hline
\end{tabular}

Table 4.

\begin{tabular}{|l|l|l|l|}
\hline$*$ & 0 & 1 & 2 \\
\hline 0 & 0 & 0 & 0 \\
\hline 1 & 1 & 0 & 1 \\
\hline 2 & 1 & 1 & 0 \\
\hline
\end{tabular}

It satisfies the condition (1) (2) (3) of the BCK definition, but doesn't the condition (4).

Example 4. Let $X=\{0,1,2\}$ and $*$ is given by the table 4

It satisfies the condition (1) (3) (4) of the BCK definition, but doesn't the condition (2).

Example 5. Let $X=\{0,1,2\}$ and $*$ is given by the table 5 . 
Table 5.

\begin{tabular}{|l|l|l|l|}
\hline$*$ & 0 & 1 & 2 \\
\hline 0 & 0 & 0 & 0 \\
\hline 1 & 2 & 0 & 0 \\
\hline 2 & 2 & 2 & 0 \\
\hline
\end{tabular}

Table 6.

\begin{tabular}{|l|l|l|l|}
\hline$*$ & 0 & 1 & 2 \\
\hline 0 & 0 & 0 & 0 \\
\hline 1 & 1 & 0 & 0 \\
\hline 2 & 2 & 1 & 0 \\
\hline
\end{tabular}

It satisfies the condition (1) (3) (4) of the BCK definition, but doesn't the condition (2).

Example 6. Let $X=\{0,1,2\}$ and $*$ is given by the table 6 .

It satisfies the condition (2) (3) (4) of the BCK definition, but doesn't the condition (1).

\section{Simplification of BCK and BCI definition}

We give two theorems, which simplify the BCK and BCI definitions by giving new equivalent conditions.

Theorem 1. Both conditions

$$
\begin{aligned}
& F_{1}: t *((((x * y) *(x * z)) *(z * y)) * s)=t \\
& F_{2}: x * y=0,
\end{aligned}
$$

$$
y * x=0 \Rightarrow x=y, \forall x, y, z, s, t \in A
$$

are equivalent to conditions (1) (2) (3) and (4) of BCK definition.

Proof: $(\Rightarrow)$ Condition $F_{2}$ implies condition (4) trivially.

Let $x=y \in A$, then $x * y=0$, that is $x * x=0$.

So when $x=y=z \in A$, we have

$$
\begin{aligned}
& ((x * y) *(x * z)) *(z * y)=((x * x) *(x * x)) *(x * x) \\
& =(0 * 0) * 0=0 .
\end{aligned}
$$

$t *(0 * 0)=t * 0=t$. According to the arbitrariness of $t$, we get that $t * 0=t \forall t \in A$, that is the condition (2) in the BCK definition.

Let $x=y=z \in A, \forall s \in A$, and assume $t=(0 * s)$, then

$$
\begin{gathered}
t *((((x * y) *(x * z)) *(z * y)) * s) \\
=(0 * s) *(0 * s)=t=(0 * s)
\end{gathered}
$$

Since $x * x=0$, we get that $(0 * s) *(0 * s)=0$, that is $0 * s=0$. By the arbitrariness of $s$, we get that $0 * s=0, \forall s \in A$, that is the condition (3) in the BCK definition.

$\forall x, y, z \in A$, let $s=0$,

$$
t=(((x * y) *(x * z)) *(z * y))
$$

then

$$
t *((((x * y) *(x * z)) *(z * y)) * s) \begin{array}{r}
=(((x * y) *(x * z)) *(z * y)) \\
*((((x * y) *(x * z)) *(z * y)) * 0)
\end{array}
$$




$$
\begin{gathered}
=(((x * y) *(x * z)) *(z * y)) \\
*(((x * y) *(x * z)) *(z * y))
\end{gathered}=0=t=(((x * y) *(x * z)) *(z * y))
$$

By the arbitrariness of $x, y, z$, we get that

$$
(((x * y) *(x * z)) *(z * y))=0, \forall x, y, z \in A,
$$

that is the condition (1) in the BCK definition.

$(\Leftarrow)$ According to the definition of BCK, we have $\forall x, y, z, s, t \in A$,

$$
\begin{gathered}
t *((((x * y) *(x * z)) *(z * y)) * s) \\
=t *(0 * s)=t * 0=t,
\end{gathered}
$$

then the condition $F_{1}$ holds.

By the condition (4) in the BCK definition, we have:

$$
x * y=0 \text { and } y * x=0 \text {, }
$$

that is $x=y$.

In order to proof the condition $F_{2}$, we only need to proof that

$$
x=y \Rightarrow x * y=0 \text { and } y * x=0,
$$

that is $x * x=0 . \forall x \in A$, let $y=z=0$,

According to the equality

$$
(((x * y) *(x * z)) *(z * y))=0
$$

we have

$(((x * 0) *(x * 0)) *(0 * 0))=(x * x) * 0=(x * x)=0$, that is $x * x=0, \forall x \in A$. Then the condition $F_{2}$ holds. Therefore, the conditions $F_{1}$ and $F_{2}$ are equivalent to the BCK definition.

Theorem 2. Both the conditions

$M_{1}: t *(((x * y) *(x * z)) *(z * y))=t, \forall t, x, y, z \in A M_{2}: x * y=0, y * x=0 \Leftrightarrow x=y, \forall x, y \in A$

are equivalent to the BCI definition.

Proof: $(\Rightarrow)$ By $M_{2}$, the condition (3) in the BCI definition is trivial.

Since $x=y, x * y=0$, we get that

$$
\forall x \in A, x * x=0
$$

Let $x=y=z \in A$, then

$$
\begin{aligned}
& (((x * y) *(x * z)) *(z * y)) \\
& \quad=(((x * x) *(x * x)) *(x * x))=((0 * 0) * 0)=0
\end{aligned}
$$

So let $x=y=z \in A, \forall t \in A$,

$$
\begin{aligned}
M_{1} & =t *(((x * y) *(x * z)) *(z * y)) \\
& =t * 0=t, \forall t \in A
\end{aligned}
$$

Then the condition (2) in the BCI definition holds.

$\forall x, y, z \in A$, let $t=(((x * y) *(x * z)) *(z * y))$, then by the condition $M_{2}$ we have:

$$
\begin{aligned}
& M_{1}: t *(((x * y) *(x * z)) *(z * y)) \\
& =(((x * y) *(x * z)) *(z * y)) \\
& \quad *(((x * y) *(x * z)) *(z * y))
\end{aligned} \quad=(((x * y) *(x * z)) *(z * y))=0 .
$$

So the condition (1) in the BCI definition holds. 
( $\Leftarrow$ ) According to the conditions (1) and (2) in the BCI definition, we have the following equality:

$$
\begin{array}{r}
t *(((x * y) *(x * z)) *(z * y)) \\
=t * 0=t, \forall x, y, z, t \in A .
\end{array}
$$

Thus the condition $M_{1}$ holds.

According to the condition (3) in the BCI definition, in order to proof the condition $M_{2}$, we only need to proof that

that is $x * x=0, \forall x \in A$.

$$
\forall x, y \in A \text {, let } x=y \text {, then } x * y=0 \text {, }
$$

Let $\forall x \in A, y=z=0$ then

$$
\begin{gathered}
(((x * y) *(x * z)) *(z * y)) \\
=(((x * 0) *(x * 0)) *(0 * 0)) \\
=((x * x) * 0)=(x * x)=0
\end{gathered}
$$

According to the arbitrariness of $x$, the condition $M_{2}$ holds.

Therefore, the conditions $M_{1}$ and $M_{2}$ are equivalent to the BCI definition.

\section{Conclusions}

We put out four examples in order to prove that any one of the four conditions in the definition of BCK algebra cannot be proofed by other three conditions. Next we simplify the definitions of the BCK and BCI Algebra by giving new equivalent conditions..

\section{Acknowledgment}

This work described here is partially supported by the National Natural Science Foundation of China (19801016) (10261003) and the Fundamental Research Funds for the Central Universities (3142013021) and the North China institute of Science and Technology Key Discipline Construction Project (HKXJZD201402).

\section{References}

[1] F. Marty, Sur une generalization de la notion de groups, 8th congress Math. Scandinaves, Stockhholm (1934) 45-49.

[2] Jie Meng, Yonglin Liu, The introduction of Algebra, Shaanxi Scientific and Technological Press . (2001) 250-258.

[3] R. Cigno li, Glivenko like theorems in natural expansions of BCK-logic, Math. Log . Quart (2) (2004) 111-125.

[4] Y. Imai and K. Iséki, On axiom systems of propositional calculi XIV, Proc. Japan Academy (1966) 19-22.

[5] Y. L. Liu, J. Meng, Fuzzy ideals in BCI-algebras, Fuzzy Sets and Sy stems (2001) 227-237. 